\title{
Telephone or Visit-Based Community Health Worker Care Management for Uncontrolled Diabetes Mellitus: A Longitudinal Study
}

\author{
Barbara J. Turner ${ }^{1,4}$ (D) Yuanyuan Liang ${ }^{2} \cdot$ Ambili Ramachandran $^{3} \cdot$ Ramin Poursani $^{3}$
}

Published online: 29 May 2020

(c) Springer Science+Business Media, LLC, part of Springer Nature 2020

\begin{abstract}
Community health workers (CHWs) can reduce health disparities for low income patients but type of contact and outcomes has had limited study. Low-income Hispanic primary care patients with hemoglobin A1c [HbA1c] $\geq 9 \%$ received care managment (CM) over 6 months classified as: (CM1) telephone only; (CM2) clinic visit but no calls; (CM3) clinic visit with calls; and (CM4) $\geq 2$ visits \pm calls. Type of CM delivery and time to DM control (HbA1c $<9 \%)$ examined in Cox proportional hazards model and more rapid control within 6 months using logistic regression. Models adjusted for demographics, clinical, and health care variables. At baseline, 523 patients had mean $\mathrm{HbA1c} 10.9 \%(\mathrm{SD}=1.7 \%)$, mean age 57.9 years $(\mathrm{SD}=10), 58.5 \%$ women, 87.6\% Hispanic, and 55.5\% uninsured. CM types for patients: 51 (9.8\%) CM1; 192 (36.7\%) CM2; 44 (8.4\%) CM3; and $236(45.4 \%)$ CM4. Median time to HbA1c control was 197 days (95\% CI [71, 548]) and 41.5\% achieved control within 6 months. Compared with CM1, control was more rapid for CM2 (Hazard ratio [HR] 1.45, 95\% CI [1.01, 2.09], p = 0.043) and CM4 but not significant (HR [95\% CI] 1.29 [0.91, 1.83], $\mathrm{p}=0.15)$. Adjusted odds of more rapid control within 6 months were twofold higher for CM2 $(p=0.04)$ and CM4 $(p=0.055)$, respectively, versus CM1. CM2 did not differ from CM1. DM control was less likely for CM by telephone only than face-to-face in clinic. To benefit vulnerable patients with uncontrolled $\mathrm{DM}$, in-person engagement may be required.
\end{abstract}

Keywords Diabetes mellitus $\cdot$ Community health worker $\cdot$ Hispanic $\cdot$ Care management $\cdot$ Telephone counseling

Presented in part at the 41st Annual Meeting of the Society of General Internal Medicine, Denver CO, April 12, 2018.

Barbara J. Turner

barbara.turner@med.usc.edu

1 Department of Medicine, Keck Medical Center of University of Southern California, 2020 Zonal Avenue, Los Angeles, CA 90033, USA

2 Department of Epidemiology and Public Health, University of Maryland School of Medicine, 660 W. Redwood Street, Baltimore, MD 21201, USA

3 Department of Medicine, Joe R. and Teresa Lozano Long School of Medicine, University of Texas Health Science Center at San Antonio (UT Health San Antonio), 7703 Floyd Curl Drive, San Antonio, TX 78229, USA

4 Gehr Center for Health Systems Science and Innovation, Keck School of Medicine of USC, 2020 Zonal Avenue, IRD 322, Los Angeles, CA 90033, USA

\section{Introduction}

Lifetime risk of diabetes mellitus for Hispanic men and women in the U.S. exceeds $50 \%$ which far exceeds that for the non-Hispanic white population [1]. Once diagnosed with diabetes, Hispanics experience greater morbidity from complications and mortality [2]. Successful management of diabetes requires patient education and supportive resources to promote treatment adherence and healthier lifestyle. Greater social support has been associated with a lower risk of diabetes among Hispanics of diverse backgrounds [3] and greater self-efficacy to manage diabetes in some Hispanic groups [4]. Community health workers (CHWs) serve a key support role as they come from similar communities and understand challenges of managing diabetes. CHWs address limited health literacy, needed resources and care, and behavioral change $[5,6]$.

A systematic review of randomized controlled trials of CHW support and education for diabetes reported improvement in patient-reported outcomes such as knowledge, self-care behaviors, emotional distress/well-being, and 
medication adherence [7]. A narrative review of randomized trials reported modest improvement in control with CHW care management as assessed by hemoglobin A1c (HbA1c), especially for patients with higher baseline levels [8]. Yet most interventions were face-to-face and this review deemed the benefit of telephone-based interventions as inconclusive [8]. However, face-to-face home visits are resource intensive and office visits can be challenging to coordinate with clinician visits. Phone calls may be more readily implemented in practices with high demand for patient support.

This study examines a pilot CHW project undertaken for a Texas' Transformation and Quality Improvement Program (1115 Medicaid waiver) project in urban primary care practices serving primarily Hispanic patients. The project aimed to improve both diabetes and hypertension control among patients with diabetes. Patients with uncontrolled diabetes based on a HbA1c $\geq 9 \%$ were offered CHW care management in face-to-face meetings in clinic, by telephone, or both. This retrospective cohort study examines the association of the type of $\mathrm{CHW}$ contacts for care management delivered over a 6-month period and time to control based on a $\mathrm{HbA} 1 \mathrm{c}<9 \%$ as well as more rapid achievement of control within 6 months.

\section{Methods}

\section{Setting and Patients}

The 1115 Medicaid Waiver allowed the state of Texas to expand Medicaid managed care and services for the uninsured. Through this 1115 Medicaid waiver, a pilot project to improve diabetes outcomes was conducted in a family medicine and a general internal medicine residency practice serving low income Hispanic-majority communities. The waiver set $\mathrm{HbA} 1 \mathrm{c}<9 \%$ as the diabetes control goal.

For this project, an electronic medical record (EMR) registry was created for patients aged 18-75 years and diagnosed with diabetes at one inpatient or two outpatient encounters (ICD-9-CM 250.xx or ICD-10 E10.xx or E11. $\mathrm{xx}$ ) or $\mathrm{HbA} 1 \mathrm{c} \geq 6.5 \%$. The registry included: demographics; diagnoses; medications; test results; and health care utilization [9]. Patients were eligible for CHW support with a $\mathrm{HbA} 1 \mathrm{c} \geq 9 \%$ or clinician referral. Eligibility criteria for this study were: most recent $\mathrm{HbA} 1 \mathrm{c} \geq 9 \%$; clinic care initiated by January 2013; and at least two clinic visits from January 2014 through December 2017 (Fig. 1). Exclusions included: no CHW encounter; fewer than two HbA1c tests; and less than 6 months in care after initiating CHW support.

\section{CHW Support and Counseling Program}

Four bilingual (Spanish or English) CHWs-also called promotoras-were certified by the Texas Department of Health and Human Services to serve in this role after completing approved training. CHWs received $20 \mathrm{~h}$ of specific training for our project on: diabetes epidemiology; complications; medications; treatment goals; and evidence-based medication adherence supports. CHWs also received print and electronic information (Spanish and English) to share with patients, family, and caregivers about community resources, affordable healthy diet for diabetes, and physical activities. CHWs reviewed and practiced motivational interviewing skills to promote patient-centered care. CHWs met weekly with clinic directors and the research team to review successes and challenges.

CHWs were charged with contacting all eligible patients by telephone and/or in clinic. Clinic contact was attempted whenever time and space were available. The 15-30 min CHW-patient meetings addressed: access to prescribed medications/devices (e.g. glucose monitor); medication adherence; access to a local health care financial assistance program; and lifestyle changes. CHWs attempted to follow-up with patients at least once. Staff-directed group diabetes education classes were also offered. The $\mathrm{CHW}$ or a research staff member recorded all patient encounters and type (phone or visit) in a REDCap database. This analysis considered only telephone calls personally with the patient while calls with family members or visit reminder messages were not.

\section{Study Variables}

\section{Dependent Variables}

The primary outcome was time from initiating CHW support until the first $\mathrm{HbA} 1 \mathrm{c}<9 \%$. A secondary outcome more rapid achievement of control as demonstrated by a HbA1c $<9 \%$ within 6 months after $\mathrm{CHW}$ initiation (Yes/No).

\section{Independent Variables}

Type of CHW care management [CM] delivered over 6 months was classified as: CM1-no visits and one or more telephone calls; $\mathrm{CM} 2$ - one visit but no calls; CM3one visit and one or more calls; and CM4-two or more visits with or without calls. Baseline data from the registry 


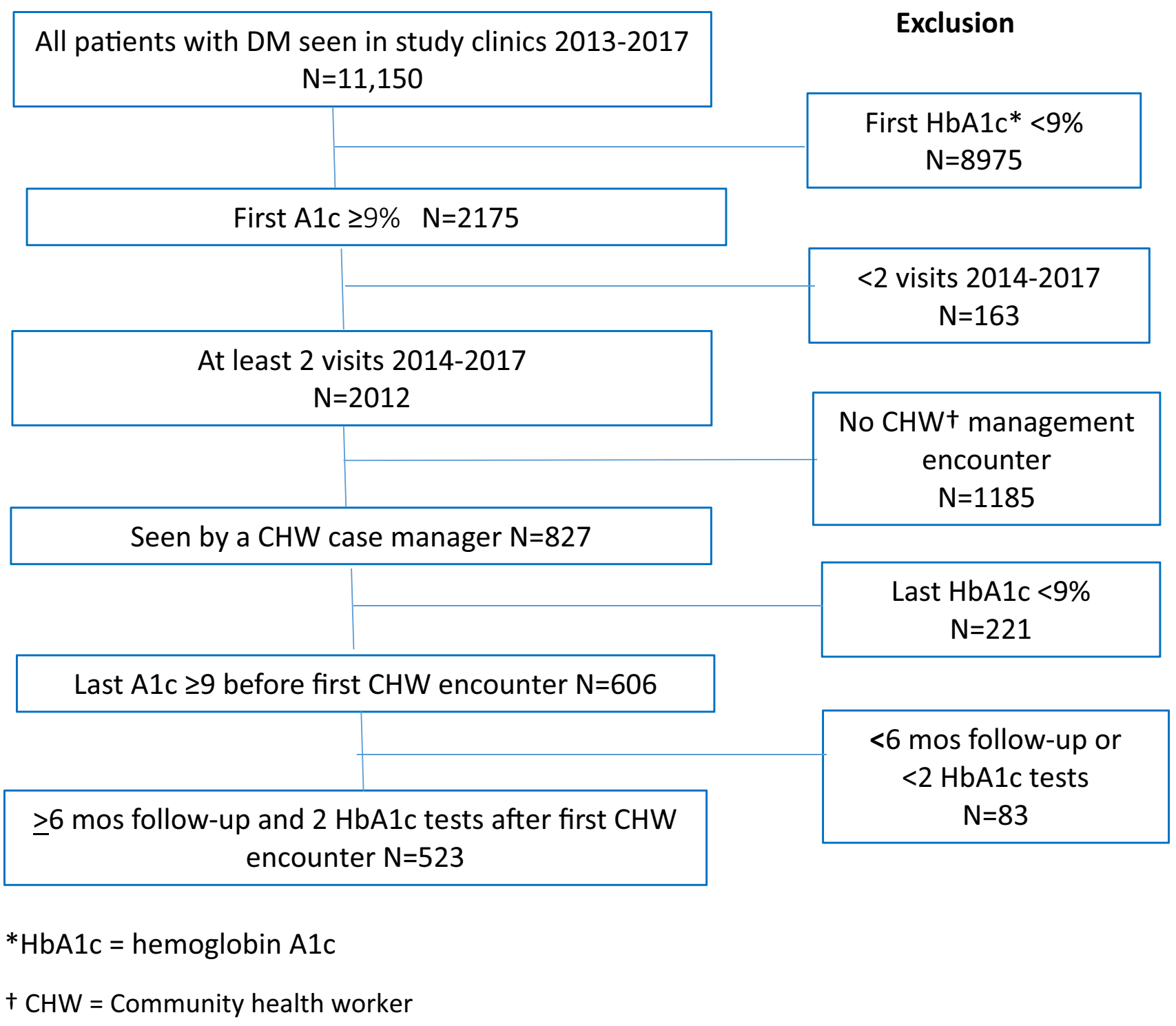

Fig. 1 Diabetes mellitus (DM) study population flow chart diabetes mellitus (DM) study population flow chart

before CM initiation included: demographics (age, race/ ethnicity, and sex); clinical (Elixhauser comorbidity score [10], most recent HbA1c, and diabetes medications/dose), and health care (insurance, primary care provider (PCP) visits in prior 6 months, offer of diabetes group visit). During the study years, available diabetes medications for these low income patients were insulin and/or four classes of oral diabetes drugs: biguanides (e.g., metformin), sulfonylureas (e.g., glimepiride); thiazolidinediones (e.g., pioglitazone), and dipeptidyl peptidase IV inhibitors (e.g., sitagliptin). With the guidance of a pharmacologist, dose of insulin (units/d) was classified as four levels (none; low < 50; medium 50-99; or high $\geq 100$ ). Dose of each oral diabetes medication was assigned a point score as: minimum (1); minimum to less than maximum (2); or maximum (3) These points were totaled for a maximum of three oral drugs (range 1-9) and categorized as: none, low (1-3), medium (4-6), or high (7-9).

\section{Statistical Analyses}

We examined patient demographic and clinical characteristics by type of CM using the chi-squared test or Fisher's exact test for categorical variables and Kruskal-Wallis $\mathrm{H}$ test or ANOVA F test for continuous variables. Kaplan-Meier curves of time to first $\mathrm{HbA} 1 \mathrm{c}<9 \%$ by the four types of $\mathrm{CM}$ were plotted and compared using the log-rank test. For the primary outcome, Cox proportional hazards model examined 
Table 1 Baseline demographic characteristics of patients with uncontrolled diabetes by type of community health worker support

\begin{tabular}{|c|c|c|c|c|c|c|}
\hline \multirow[t]{2}{*}{ Demographic characteristics } & \multirow[t]{2}{*}{ All patients } & \multicolumn{4}{|c|}{ Community Health Worker Care Management (CM) } & \multirow[t]{2}{*}{$\mathrm{P}$ value } \\
\hline & & $\begin{array}{l}\text { CM } 1 \\
\text { (No visit; } 1+\text { calls) }\end{array}$ & $\begin{array}{l}\text { CM } 2 \\
\text { (1 visit, no calls) }\end{array}$ & $\begin{array}{l}\text { CM } 3 \\
\text { (1 visit; } 1+\text { calls })\end{array}$ & $\begin{array}{l}\mathrm{CM} 4 \\
(2+\text { visits }, \pm \text { calls })\end{array}$ & \\
\hline Total, n (\%) & 523 & $51(9.8)$ & $192(36.7)$ & $44(8.4)$ & $236(45.1)$ & \\
\hline Age, years (mean) & $57.9(10)$ & $57.2(9.1)$ & $58.7(10.1)$ & $58.8(8.8)$ & $57.3(10.4)$ & 0.46 \\
\hline Women, n (\%) & $306(58.5)$ & $28(54.9)$ & $105(54.7)$ & $26(59.1)$ & $147(62.3)$ & 0.42 \\
\hline Race-ethnicity, n (\%) & & & & & & 0.20 \\
\hline Hispanic & $458(87.6)$ & $41(80.4)$ & $173(90.1)$ & $39(88.4)$ & $205(86.9)$ & \\
\hline Non-Hispanic white & $34(6.5)$ & $8(15.7)$ & $9(4.7)$ & $1(2.3)$ & $16(6.8)$ & \\
\hline Non-Hispanic black & $21(4)$ & $2(3.9)$ & $5(2.6)$ & $3(6.8)$ & $11(4.7)$ & \\
\hline Other & $10(1.9)$ & $0(0)$ & $5(2.6)$ & $1(2.3)$ & $4(1.7)$ & \\
\hline Insurance, n (\%) & & & & & & 0.07 \\
\hline Insured & $46(8.8)$ & $7(13.7)$ & $7(3.65)$ & $3(6.8)$ & $29(12.3)$ & \\
\hline Medicaid & $56(10.7)$ & $5(9.8)$ & $27(14.1)$ & $2(4.6)$ & $22(9.3)$ & \\
\hline Medicare & $133(25.4)$ & $14(27.5)$ & $48(25.0)$ & $11(25.0)$ & $60(25.4)$ & \\
\hline Uninsured & $288(55.1)$ & $25(49.0)$ & $110(57.3)$ & $28(63.4)$ & $125(53.0)$ & \\
\hline
\end{tabular}

the association of type of $\mathrm{CM}$ with time to first $\mathrm{HbA} 1 \mathrm{c}<9 \%$ adjusted for all available important demographic and clinical variables. For the secondary outcome, the association of type of $\mathrm{CM}$ with achieving an $\mathrm{HbA} 1 \mathrm{c}<9 \%$ within 6 months after CM initiation was examined using logistic regression and adjusted for the same covariates. Statistical analyses were performed using Stata/SE 16 (College Station, TX).

Table 2 Baseline clinical characteristics by diabetes mellitus (DM) case management typology

\begin{tabular}{|c|c|c|c|c|c|c|}
\hline \multirow[t]{2}{*}{ Clinical characteristics at baseline } & \multicolumn{6}{|c|}{ Diabetes case management $(\mathrm{CM})$ typology } \\
\hline & All patients & $\begin{array}{l}\text { CM } 1 \\
\text { (No visit; } 1+\text { calls) }\end{array}$ & $\begin{array}{l}\text { CM } 2 \\
\text { (1 visit; no calls) }\end{array}$ & $\begin{array}{l}\text { CM } 3 \\
\text { (1 visit; } 1+\text { calls })\end{array}$ & $\begin{array}{l}\text { CM } 4 \\
(2+\text { visits }, \pm \text { calls })\end{array}$ & $P$ value \\
\hline Total, n & 523 & 51 & 192 & 44 & 236 & \\
\hline Hemoglobin A1c, \% mean (SD) & $10.9(1.7)$ & $10.8(1.6)$ & $10.9(1.8)$ & $11.2(1.6)$ & $10.9(1.7)$ & 0.75 \\
\hline Elixhauser comorbidity score, mean (SD) & $4(2.4)$ & $4.6(2.3)$ & $4.1(2.4)$ & $4.1(2.3)$ & $3.8(2.3)$ & 0.01 \\
\hline Type of DM medication & & & & & & 0.67 \\
\hline Insulin only & $127(24.3)$ & $9(17.7)$ & $54(28.1)$ & $10(22.7)$ & $54(22.9)$ & \\
\hline Oral only & $152(29.1)$ & $16(31.4)$ & $54(28.1)$ & $14(31.8)$ & $68(28.8)$ & \\
\hline Both & $172(32.9)$ & $17(33.3)$ & $54(28.1)$ & $15(34.1)$ & $86(36.4)$ & \\
\hline Neither & $72(13.8)$ & $9(17.7)$ & $30(15.6)$ & $5(11.4)$ & $28(11.9)$ & \\
\hline Total oral DM medication dose* N (\%) & & & & & & 0.55 \\
\hline None & $199(38.1)$ & $18(35.3)$ & $84(43.8)$ & $15(34.1)$ & $82(34.8)$ & \\
\hline Low & $80(15.3)$ & $9(17.7)$ & $26(13.5)$ & $4(9.1)$ & $41(17.4)$ & \\
\hline Moderate & $157(30)$ & $15(29.4)$ & $54(28.1)$ & $18(40.9)$ & $70(29.7)$ & \\
\hline High & $87(16.6)$ & $9(17.7)$ & $28(14.6)$ & $7(15.9)$ & $43(18.2)$ & \\
\hline Insulin $\operatorname{dose}^{\dagger} \mathrm{N}(\%)$ & & & & & & 0.94 \\
\hline None & $224(42.8)$ & $25(49)$ & $84(43.8)$ & $19(43.2)$ & $96(40.7)$ & \\
\hline Low & $145(27.7)$ & $11(21.6)$ & $49(25.5)$ & $13(29.6)$ & $72(30.5)$ & \\
\hline Moderate & $66(12.6)$ & $5(9.8)$ & $25(13)$ & $5(11.4)$ & $31(13.1)$ & \\
\hline High & $88(16.8)$ & $10(19.6)$ & $34(17.7)$ & $7(15.9)$ & $37(15.7)$ & \\
\hline $\begin{array}{l}\text { Primary care visits in prior } 6 \text { months, } \\
\text { mean (SD) }\end{array}$ & $3.2(2.3)$ & $3.4(2.2)$ & $3.6(2.4)$ & $2.9(2.1)$ & $3.0(2.3)$ & 0.05 \\
\hline Offered DM group education, N (\%) & $290(56)$ & $27(53)$ & $116(60)$ & $24(55)$ & $123(52)$ & 0.37 \\
\hline
\end{tabular}

*See methods: dose for all oral DM drugs (range 1-9) was totaled and categorized in four levels: none, low (1-3), moderate (4-6), and high (7-9)

${ }^{\dagger}$ Dose of insulin (units/d) at baseline classified as four levels: none; low $<50$; medium 50-99; or high $>100$ 


\section{Compliance with Ethical Standards}

The authors declare that they have no conflicts of interest. The Institutional Review Boards of both University of Texas Health Science Center at San Antonio and University Health System (UHS) approved the study and judged this quality improvement study as exempt from patient consent. The funding agency had no role in conducting or analyzing the results of this study.

\section{Results}

The study cohort included 523 subjects with a mean age of 57.9 years $(\mathrm{SD}=10)$ and $58.5 \%$ women (Table 1$)$. Most subjects were Hispanic (87.6\%) and over half $(55.1 \%)$ were uninsured and paid for care through a local financial assistance program. The median observation time for this cohort was 626 [first quartile $=393$, third quartile $=935$ ] days.

In regard to type of $\mathrm{CM}$ received over 6 months, 236 (45.1\%) subjects received at least two office visits with or without telephone calls (CM4). One office visit with a CHW but no telephone calls (CM2) was received by 192 subjects $(36.7 \%)$ while only telephone calls (CM1) or one visit with telephone calls (CM3) was received by 51 (9.8\%) and 44 (8.4\%) subjects, respectively. Type of CM received was not significantly associated with patient demographics.

Mean baseline $\mathrm{HbA} 1 \mathrm{c}$ was $10.9 \%(\mathrm{SD}=1.7 \%)$ and mean Elixhauser comorbidity score was $4(\mathrm{SD}=2.4)$ (Table 2$)$. Oral diabetes medication without insulin was prescribed for $29.1 \%$ of subjects, $24.3 \%$ received only insulin, and onethird received oral drugs with insulin. Only $13.8 \%$ of subjects were not treated at baseline. Among subjects receiving oral diabetes drugs, the most frequent dose category was medium but, among subjects treated with insulin, the most frequent dose was low. Significant differences by type of CM included higher comorbidity score for CM1 subjects and more prior $\mathrm{PCP}$ visits for $\mathrm{CM} 1$ and $\mathrm{CM} 2$ subjects.

Both CM2 (one visit, no calls) and CM4 (two visits or more visits, with or without calls) were associated with a shorter time to diabetes control than CM1 (only calls) or CM3 (one visit and calls), log-rank $\mathrm{p}=0.053$ (Fig. 2). Not shown, median time (days) to control was: 350 days for CM1; 159 days for CM2; 281 days for CM3; and 197 days for CM4. Compared with CM1 (reference), the adjusted hazard ratio for diabetes control for CM2 was $1.45(\mathrm{p}=0.043)$ and 1.29 for CM4 but not significant ( $\mathrm{p}=0.15$ ). CM3 did not differ from CM1 (Table 3). The Cox model also showed that

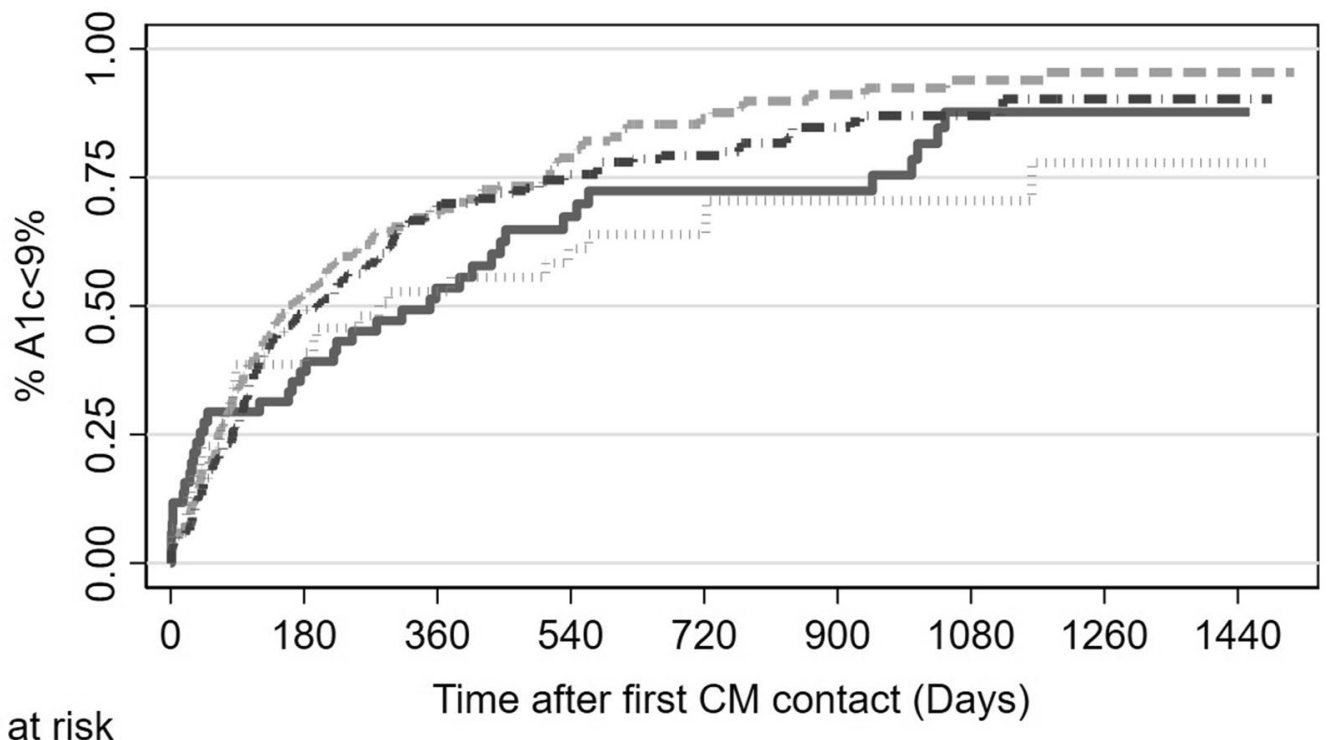

Number at risk

\begin{tabular}{|c|c|c|c|c|c|c|c|c|}
\hline \multirow{2}{*}{$\overline{5-1}$} & \multirow{2}{*}{$\begin{array}{c}51 \\
192\end{array}$} & & & & & & & \multirow[b]{2}{*}{3} \\
\hline & & $\begin{array}{l}32 \\
88\end{array}$ & $\begin{array}{l}22 \\
54\end{array}$ & $\begin{array}{l}13 \\
27\end{array}$ & $\begin{array}{l}10 \\
12\end{array}$ & $\begin{array}{l}9 \\
7\end{array}$ & $\begin{array}{l}4 \\
4\end{array}$ & \\
\hline & 44 & 26 & 18 & 14 & 11 & 4 & 4 & 2 \\
\hline - & 236 & 120 & 65 & 43 & 28 & 15 & 8 & 2 \\
\hline & & & & 1 vis & & & & \\
\hline
\end{tabular}

Fig. 2 Kaplan-Meier curves for time to first hemoglobin A1c $<9 \%$ after case management initiation for patients categorized by four case management typologies 
Table 3 Cox model for time from initiating diabetes mellitus (DM) case management to first hemoglobin A1c control <9\%

\begin{tabular}{llll}
\hline Variable & Hazard ratio & $95 \%$ CI & P value \\
\hline $\begin{array}{l}\text { Case management (CM) typol- } \\
\text { ogy }\end{array}$ & & & \\
CM 2 (1 visit) & 1.45 & $1.01-2.09$ & 0.043 \\
CM 3 (1 visit + calls) & 0.98 & $0.60-1.59$ & 0.93 \\
CM 4 (2 visits \pm calls) & 1.29 & $0.91-1.83$ & 0.154 \\
Elixhauser score (per comorbid- & 0.95 & $0.91-1.00$ & 0.044 \\
$\quad$ ity) & & & \\
Total oral DM medication dose ${ }^{\dagger}$ & & & \\
Low & 0.78 & $0.57-0.89$ & 0.104 \\
Moderate & 0.72 & $0.56-0.92$ & 0.008 \\
High & 0.71 & $0.53-0.96$ & 0.030 \\
Insulin dose & & & \\
Low & & & \\
Moderate & 0.70 & $0.55-0.89$ & 0.004 \\
High & 0.84 & $0.61-1.15$ & 0.27 \\
Primary care visits in 6 mos & 0.72 & $0.54-0.97$ & 0.030 \\
before CM & & & \\
$1-2$ & 0.70 & $0.49-0.99$ & 0.046 \\
3-4 & 0.96 & $0.67-1.38$ & 0.84 \\
5+ & 0.92 & $0.64-1.35$ & 0.74 \\
\hline
\end{tabular}

Reference groups: CM 1 (calls), uninsured, no insulin, no oral diabetes medication, no primary care visits before $\mathrm{CM}$; Also adjusted for age, sex, Hispanic ethnicity, baseline A1c, insurance type, offer group DM education

†See methods: dose for all oral DM drugs (range 1-9) was totaled and categorized in four levels: none, low (1-3), moderate (4-6), and high (7-9)

${ }^{\ddagger}$ Dose of insulin (units/d) at baseline classified as four levels: none; low $<50$; medium 50-99; or high $>100$

a longer time to control $(\mathrm{p}<0.05)$ was required for: greater comorbidity; higher dose oral diabetes medication; low or high dose insulin; and 1-2 primary care visits in past six months versus none.

By 6 months after CM initiation, the entire cohort had a mean $\mathrm{HbA} 1 \mathrm{c}$ reduction of $-1.37 \%(\mathrm{SD}=2.2 \%)$ but the reduction was even greater for $\mathrm{CM} 2(-1.58 \%$ [SD $=2.33 \%]$, not shown). Overall, $41.5 \%$ of the cohort achieved more rapid diabetes control by 6 months. Adjusted odds of control by 6 months were approximately twofold higher for CM2 $(p=0.046)$ and CM4 $(p=0.055)$ versus CM1 (Table 4). Again, CM3 did not differ from CM1. Rapid control within 6 months was negatively associated with greater comorbidity $(p=0.001)$ and low dose insulin $(p=0.05)$. Five or more PCP visits within the 6 months prior to CM initiation was associated with over two-fold higher adjusted odds of achieving rapid control versus none $(\mathrm{p}=0.016)$.

\section{Discussion}

In a systematic review examining multiple chronic conditions, CHW care management (CM) was reported to be cost effective for low-income, underserved patients but the specific types of CHW contact were not assessed [11]. Among 523 primarily Hispanic primary care subjects with poor diabetes control, this 3-year observational pilot study suggests that CHW contact only by telephone (CM1) was less likely to achieve control over time compared with face-to-face meetings (CM2 and CM4). This difference translated into a median of more than 150 days longer to achieve control for subjects receiving CM1 (350 days) than for those with either CM2 and CM4 (both less than 200 days). Our study also found that patients who received either CM2 or CM4 had approximately two-fold higher adjusted odds of achieving control more rapidly within 6 months after $\mathrm{CM}$ initiation as compared to CM1.

Other studies have observed less benefit from telephone CHW support. A review of telephone-based diabetes management by lay health workers judged the evidence to be weak [12]. A randomized trial of telephone-based diabetes counseling by CHWs in a local health department reported no improvement in HbA1c control among low income subjects [13]. CHW support may also require individualized in-person encounters; a randomized trial of group-based diabetes education by $\mathrm{CHW}$ for Hispanics with uncontrolled diabetes did not reduce $\mathrm{HbA1c}$ [14].

The significant improvement among subjects with one face-to-face $\mathrm{CHW}$ clinic visit compared with those receiving telephone contacts remained after adjusting for diverse demographics, treatment, and health care variables. It is possible that these individuals required only limited support to address their barriers to following the treatment plan. Future studies should aim to identify characteristics of patients with uncontrolled diabetes who might not require more intensive face-to-face $\mathrm{CHW}$ support because $\mathrm{CHW}$ interventions have usually involved numerous encounters. A trial of four CHW home visits with an average of 20 telephone calls achieved a significant reduction in HbA1c [15]. Similarly, up to 36 home visits by CHWs plus calls significantly reduced HbA1c among Mexican-American subjects at 1 and 2 years follow-up [16]. Among subjects with mean baseline HbA1c 9.6\%, a CHW intervention of up to 17 visits reduced mean HbA1c by $0.47 \%$ at 6 months versus controls and was maintained after 12 and 18 months [17]. However, the sustainability of CHW support for low income primary care patients requires attention to the infrastructure required for in-person clinic encounters including space, $\mathrm{CHW}$ time, and systems to encourage patients to take advantage of this resource.

Of note, our study subjects had markedly poor diabetes control with a baseline mean $\mathrm{HbA1c}$ of $10.9 \%$; unfortunately, 
Table 4 Logistic regression model of achieving hemoglobin A1c control $<9 \%$ within 6 months after diabetes mellitus (DM) case management initiation

\begin{tabular}{llll}
\hline Variable & Odds ratio & $95 \%$ CI & P value \\
\hline Case management (CM) typology & & & \\
CM 2 (1 visit) & 2.08 & $1.01-4.27$ & 0.046 \\
CM 3 (1 visit + calls) & 1.08 & $0.42-2.79$ & 0.88 \\
CM 4 (2 visits +calls) & 1.99 & $0.99-4.03$ & 0.055 \\
Elixhauser score (per comorbidity) & 0.85 & $0.77-0.93$ & 0.001 \\
Total oral DM medication dose ${ }^{\dagger}$ & & & 0.25 \\
Low & 1.40 & $0.78-2.50$ & 0.40 \\
Moderate & 0.81 & $0.51-1.31$ & 0.60 \\
High & 1.16 & $0.67-2.03$ & \\
Insulin dose ${ }^{\ddagger}$ & & & 0.05 \\
Low & 0.63 & $0.40-1.00$ & 0.33 \\
Moderate & 0.74 & $0.40-1.36$ & 0.11 \\
High & 0.63 & $0.36-1.11$ & \\
Primary care visits in 6 months before CM & & & 0.28 \\
1-2 & 0.69 & $0.35-1.35$ & 0.28 \\
$3-4$ & 1.45 & $0.74-2.82$ & 0.016 \\
$5+$ & 2.34 & $1.17-4.69$ & \\
\hline
\end{tabular}

Reference groups: CM 1 (calls), no insulin, no oral DM medication, no primary care visits before CM Also adjusted for age, sex, Hispanic ( $\mathrm{Y} / \mathrm{N})$, baseline A1c, insurance type, offer group DM education

†See methods: dose for all oral DM drugs (range 1-9) totaled and categorized in four levels: none, low (1-3), moderate (4-6), and high (7-9)

${ }^{\ddagger}$ Dose of insulin (units/d) at baseline classified as four levels: none; low $<50$; medium 50-99; or high $>100$ this is common among Hispanics in the US. Not only is the age-standardized prevalence of diabetes in Hispanics over twice that of non-Hispanic whites [18], but, nationally, less than half of Hispanics with diabetes achieved a HbAlc $<7 \%$ [19]. Gratifyingly, the mean reduction in $\mathrm{HbA1c}$ for our entire cohort was $-1.37 \%$ supporting a clinically significant improvement but the reduction was even greater for the group with a face-to-face visit $(-1.58 \% \mathrm{HbA} 1 \mathrm{c})$.

Types of CM received did not differ significantly by subjects' demographic characteristics. However, the comorbidity score did differ and may indicate the type of subjects who need to be targeted with in-person support. Patients who received a face-to-face meeting and both phone calls (CM3) comprised only $9 \%$ of the cohort but did not significantly improve versus CM1. It is possible these patients did not accept another meeting in clinic because they did not want CHW help. The favorable association of CM4, the group with multiple visits with or without calls, was not significant in the time to event analysis but was associated with nearly two-times greater adjusted odds of control within 6 months.

Among our study limitations, foremost is our observational design that cannot prove causality. However, our analyses adjusted for multiple factors that can influence achievement of control. Second, the funding agency set HbA1c $<9 \%$ for diabetes control, similar to other studies in Medicaid enrollees [20]. Yet, a reduction of HbA1c to $<9 \%$ would not protect against diabetes-related complications [21]. Still, the reduction in HbA1c at 6 months for our cohort $(-1.38 \%)$ was greater than the mean $-0.45 \%$ observed for another CHW intervention in Hispanics [22]. Third, although type and intensity of diabetes medications prescribed at baseline were significantly associated with achievement of control, unmeasured medication adjustments during receipt of CHW support may have also influenced outcomes. Fourth, this study was conducted in two primary care practices serving largely uninsured, Hispanic patients and may not be relevant to practices serving different populations.

These observational cohort data should galvanize further studies to inform policymakers, administrators, and clinicians to define how to take best advantage of the talents of CHW for low-income minority populations with diabetes while conserving resources. It does require practices to set aside time and space for CHWs to see patients in clinic while other more intensive models involve home visits by CHWs. If $\mathrm{CHW}$ care management for patients from low income and other vulnerable populations is to become widely available, an important research priority will also aim to identify patients most likely to benefit from this support and examine outcomes of various types and intensities of delivering CHW services.

In conclusion, most studies of telephone-based counseling for diabetes have involved nurses and report disappointing results in reducing HbA1c [23-25]. We also found limited benefit for telephone calls by CHWs for diabetes support. 
However, our longitudinal observational study offers encouraging preliminary evidence regarding the benefit of face-toface CHW support in clinic for poorly controlled Hispanic patients. Yet with advances in telemedicine related to the COVID-19 pandemic, it will be more important than ever to define more effective support strategies that do not require face-to-face encounters in primary care practices.

Funding CMS Medicaid 1115 Waiver for the Texas Healthcare Transformation and Quality Improvement Program \#085144601.1.2.2.

\section{References}

1. Narayan, K. M., Boyle, J. P., Thompson, T. J., Sorensen, S. W., \& Williamson, D. F. (2003). Lifetime risk for diabetes mellitus in the United States. JAMA, 290, 1884-1890.

2. Centers for Disease Control and Prevention. Hispanic/Latino Americans and Type 2 Diabetes. Retrieved March 5, 2020, from https://www.cdc.gov/diabetes/library/features/hispanic-diabetes. html.

3. Gallo, L. C., Fortmann, A. L., McCurley, J. L., Isasi, C. R., Penedo, F. J., Daviglus, M. L., et al. (2015). Associations of structural and functional social support with diabetes prevalence in U.S. Hispanics/Latinos: Results from the HCHS/SOL Sociocultural Ancillary Study. Journal of Behavioral Medicine, 38, 160-170.

4. Mansyur, C. L., Rustveld, L. O., Nash, S. G., \& Jibaja-Weiss, M. L. (2015). Social factors and barriers to self-care adherence in Hispanic men and women with diabetes. Patient Education and Counseling, 98, 805-810.

5. Han, H. R., McKenna, S., Nkimbeng, M., Wilson, P., Rives, S., Ajomagberin, O., et al. (2019). A systematic review of community health center based interventions for people with diabetes. Journal of Community Health, 44, 1253-1280.

6. Kim, D., Li, A. A., Perumpail, B. J., Gadiparthi, C., Kim, W., Cholankeril, G., et al. (2019). Changing trends in etiology- and ethnicity-based annual mortality rates of cirrhosis and hepatocellular carcinoma in the United States. Hepatology, 69(3), 1064-1074.

7. Trump, L. J., \& Mendenhall, T. J. (2017). Community health workers in diabetes care: A systematic review of randomized controlled trials. Families, Systems and Health, 35, 320-340.

8. Palmas, W., March, D., Darakjy, S., Findley, S. E., Teresi, J., Carrasquillo, O., et al. (2015). Community health worker interventions to improve glycemic control in people with diabetes: A systematic review and meta-analysis. Journal of General Internal Medicine, 30, 1004-1012.

9. Zullig, L. L., Liang, Y., Vale, A. S., Trevino, A., Bosworth, H. B., \& Turner, B. J. (2017). Trajectory of systolic blood pressure in a low-income, racial-ethnic minority cohort with diabetes and baseline uncontrolled hypertension. Journal of Clinical Hypertension (Greenwich), 19, 722-730.

10. Elixhauser, A., Steiner, C., Harris, D. R., \& Coffey, R. M. (1998). Comorbidity measures for use with administrative data. Medical Care, 36, 8-27.

11. Kim, K., Choi, J. S., Choi, E., Nieman, C. L., Joo, J. H., Lin, F. R., et al. (2016). Effects of community-based health worker interventions to improve chronic disease management and care among vulnerable populations: A systematic review. American Journal of Public Health, 106, e3-e28.

12. Small, N., Blickem, C., Blakeman, T., Panagioti, M., Chew-Graham, C. A., \& Bower, P. (2013). Telephone based self-management support by 'lay health workers' and 'peer support workers' to prevent and manage vascular diseases: A systematic review and meta-analysis. BMC Health Services Research, 13, 533-533.

13. Nelson, K., Taylor, L., Silverman, J., Kiefer, M., Hebert, P., Lessler, D., et al. (2017). Randomized controlled trial of a community health worker self-management support intervention among low-income adults with diabetes, Seattle, Washington, 2010-2014. Preventing Chronic Disease, 14, E15.

14. Wagner, J. A., Bermudez-Millan, A., Damio, G., Segura-Perez, S., Chhabra, J., Vergara, C., et al. (2016). A randomized, controlled trial of a stress management intervention for Latinos with type 2 diabetes delivered by community health workers: Outcomes for psychological wellbeing, glycemic control, and cortisol. Diabetes Research and Clinical Practice, 120, 162-170.

15. Carrasquillo, O., Lebron, C., Alonzo, Y., Li, H., Chang, A., \& Kenya, S. (2017). Effect of a community health worker intervention among Latinos with poorly controlled type 2 diabetes: The Miami Healthy Heart Initiative Randomized Clinical Trial. JAMA Internal Medicine, 177, 948-954.

16. Rothschild, S. K., Martin, M. A., Swider, S. M., Tumialan Lynas, C. M., Janssen, I., Avery, E. F., et al. (2014). Mexican American trial of community health workers: A randomized controlled trial of a community health worker intervention for Mexican Americans with type 2 diabetes mellitus. American Journal of Public Health, 104, 1540-1548.

17. Perez-Escamilla, R., Damio, G., Chhabra, J., Fernandez, M. L., Segura-Perez, S., Vega-Lopez, S., et al. (2015). Impact of a community health workers-led structured program on blood glucose control among Latinos with type 2 diabetes: The DIALBEST trial. Diabetes Care, 38, 197-205.

18. Cheng, Y. J., Kanaya, A. M., Araneta, M. R. G., Saydah, S. H., Kahn, H. S., Gregg, E. W., et al. (2019). Prevalence of diabetes by race and ethnicity in the United States, 2011-2016. JAMA, 322, 2389-2398.

19. Casagrande, S. S., Aviles-Santa, L., Corsino, L., Daviglus, M. L., Gallo, L. C., Espinoza Giacinto, R. A., et al. (2017). Hemoglobin A1c, blood pressure, and LDL-cholesterol control among Hispanic/Latino Adults with diabetes: Results from the Hispanic Community Health Study/Study of Latinos (HCHS/SOL). Endocrine Practice, 23, 1232-1253.

20. Hatch, B., Marino, M., Killerby, M., Angier, H., Hoopes, M., Bailey, S. R., et al. (2017). Medicaid's impact on chronic disease biomarkers: A cohort study of community health center patients. Journal of General Internal Medicine, 32, 940-947.

21. American Diabetes Association. (2020). Glycemic targets: Standards of medical care in diabetes-2020. Diabetes Care, 43, S66-S76.

22. Spencer, M. S., Kieffer, E. C., Sinco, B., Piatt, G., Palmisano, G., Hawkins, J., et al. (2018). Outcomes at 18 months from a community health worker and peer leader diabetes self-management program for Latino adults. Diabetes Care, 41, 1414-1422.

23. Eakin, E. G., Winkler, E. A., Dunstan, D. W., Healy, G. N., Owen, N., Marshall, A. M., et al. (2014). Living well with diabetes: 24-month outcomes from a randomized trial of telephone-delivered weight loss and physical activity intervention to improve glycemic control. Diabetes Care, 37, 2177-2185.

24. Edelman, D., Dolor, R. J., Coffman, C. J., Pereira, K. C., Granger, B. B., Lindquist, J. H., et al. (2015). Nurse-led behavioral management of diabetes and hypertension in community practices: 
A randomized trial. Journal of General Internal Medicine, 30, 626-633.

25. Mons, U., Raum, E., Kramer, H. U., Ruter, G., Rothenbacher, D., Rosemann, T., et al. (2013). Effectiveness of a supportive telephone counseling intervention in type 2 diabetes patients: Randomized controlled study. PLOS ONE, 8, e77954.
Publisher's Note Springer Nature remains neutral with regard to jurisdictional claims in published maps and institutional affiliations. 